\title{
An Optimization Model of the Economic Growth under Presence of Structural Changes
}

\author{
Alexander M. Tarasyev ${ }^{1,2, a)}$, Anastasiia A. Usova ${ }^{1,2, b)}$, Alexander A. Tarasyev ${ }^{2, c)}$ \\ ${ }^{1}$ Krasovskii Institute of Mathematics and Mechanics of the Ural Branch of the Russian Academy of Sciences, \\ 620990, S. Kovalevskaya str. 16, Ekaterinburg, Russia \\ ${ }^{2}$ Ural Federal University, 620002, Mira Street, 19, Ekaterinburg, Russia \\ a)tam@imm.uran.ru \\ b)ausova@imm.uran.ru \\ ${ }^{c}$ Corresponding author: alextarassiev@mail.ru
}

\begin{abstract}
The paper investigates economic growth models with non-smooth production functions. The non-smoothness of a production function can be caused, for example, by structural changes in the economy of a region (or a country). Another possible reason is the self-adjustment property of the model, which implies that estimated parameters of the production function may change after the econometric analysis conducted for statistical data augmented with data of new periods. Application of the Pontryagin maximum principle leads to analysis of the Hamiltonian system that includes derivatives of the production function, therefore, the smoothness property of the production function is important for this application. The paper proposes the technique that makes the production function differentiable and, under some additional assumptions, does not affect the qualitative behavior of optimal solutions. Theoretical results are supported by the numerical example.
\end{abstract}

Keywords: optimal control, maximum principle, Hamiltonian systems, asymptotic state observers.

\section{INTRODUCTION}

The paper considers the optimal control problem that is based on the economic growth model. In this concern, we should refer the fundamental works of the famous economists and mathematicians suggesting the methodology for constructing mathematical models, which describe interconnection of the most significant macroeconomic factors, specifically, works of K. Arrow, R. Sollow, K. Shell, G.M. Grossman, E. Helpman, R. Ayres [2, 4, 8, 14, 15]. Departing from the mathematical growth model, the optimal control problem is formulated, which is aimed for dynamic optimization of investments in increasing effectiveness of basic production factors $[6,7,9,11,19]$.

In this paper, the model identification is performed using methods of econometrics [5]. The identification procedure is focused on production functions with switching modes. These functions include dummy variables for revealing possible qualitative changes in the economy of a country. Detection of the structural changes and their treatment in dynamic optimization methods is one of the goals of the research. In the paper, we construct an approximation of the original production function, such that the approximated function has all necessary properties commonly inherent in production functions. Using the calibrated models and the approximated production function, we consider control problems on optimal distribution of investments in the capital stock of country's economy. The quality of the control process is estimated by the integral consumption index discounted on the infinite time interval $[3,12,16]$. The problem analysis, provided in the paper, is based on the Pontryagin maximum principle [13] for the problems with the infinite time horizon $[1,3,10]$. Qualitative analysis of the Hamiltonian systems within the maximum principle includes searching for stationary regimes and investigation of the Jacoby matrices evaluated at the steady states.

The paper includes numerical results for forecast scenarios of development of the Japan's economy that are constructed using the algorithms proposed in the papers $[12,17,18]$ for solving the Hamiltonian systems. In the

International Conference of Numerical Analysis and Applied Mathematics ICNAAM 2019

AIP Conf. Proc. 2293, 120006-1-120006-4; https://doi.org/10.1063/5.0027181

Published by AIP Publishing. 978-0-7354-4025-8/\$30.00 
numerical experiment, the original model considers the non-smooth production function that is glued from two functions of the Cobb-Douglas type. Using the given continuous production function, we derive its approximation by constructing an asymptotic state observer. The found estimate of the production function is used for the solution of the optimal control problem. In conclusion, the comparison of statistical trends and simulated trajectories is provided.

The next section of the paper is devoted to the description of the growth model and to the statement of the optimal control problem. Further, we conduct the analysis of the optimal control problem within the Pontryagin maximum principle. Numerical solutions and their comparison with statistical data are carried out in the last section.

\section{GROWTH MODEL AND CONTROL PROBLEM}

A model is focused on the analysis of GDP of a region (or, a country), which is defined as the market value of all final goods and services produced within a country in a year. As a production factor, we consider the value $\mathrm{k}$ of capital per worker, and the output y is taken as GDP per worker. Production function $y=f(k)$ describes the dependence of the output from the factor $\mathrm{k}$. The structure of the production function is determined in accordance with the statistical data using the econometric methods. This circumstance may result in nonsmoothness of the production function, for example, in the cases, where the econometric analysis reveals the qualitative changes in the statistical trends.

It is supposed that $y=f(k)$ satisfies the assumptions generally imposed on the production functions, namely, it is a positive, continuous, increasing and concave function for all $k \in \kappa$, where $\kappa \in(0 ;+\infty)$ is a nonempty set, which is called economic domain. If the production function is not smooth, we suppose that it is a continuous piecewise function whose pieces are positive, increasing and concave functions. Moreover, we assume that there exists a good approximation $\hat{y}=\hat{f}(k)$ of the production function $y=f(k)$ for all $k \in \kappa$. The approximated production function has the properties inherent in a standard production function, i.e. it is a positive, increasing, and concave function for all $k \in \kappa$. The dynamics of the production factor $k$ is described by the equation:

$$
\begin{aligned}
& \dot{k}(t)=u f(k(t))-\delta k, k(0)=k_{0} \in \kappa \\
& y=f(k)
\end{aligned}
$$

Here, parameter $\mathrm{u}$ is a control variable that in economic growth models is interpreted as a share of the output $y$ invested into the production factor $k$. Positive parameter $\delta$ stands for the capital depreciation rate. The right-hand part of the dynamic equation (1) is not smooth in phase variable $k$, since $y(k)$ is not smooth function. Therefore, we introduce the system of the following form:

$$
\begin{aligned}
& \dot{k}(t)=u \hat{f}(k(t))-\delta k, k(0)=k_{0} \in \kappa, \\
& \hat{y}=\hat{f}(k)
\end{aligned}
$$

where the original production function $y=f(k)$ is replaced by its smooth estimate $\hat{y}=\hat{f}(k)$.

The quality of the control is estimated by the integral consumption index of the form:

$$
\hat{J}(\cdot)=\int_{0}^{+\infty} e^{-\rho t} \ln \hat{c}(t) d t,
$$

where the consumption level $\hat{c}(t)$ can be found by the formula $\hat{c}(t)=\hat{f}(k(t))(1-u(t))$, and the positive parameter $\rho$ is the discount factor.

Under the assumption on closeness of the economic system, we suppose that control $u$ satisfies restrictions:

$$
u \in[0, \bar{u}]=: U, 0<\bar{u}<1,
$$

where $\bar{u}$ is an upper boundary for the investment share.

The problem consists in searching for a such control process $\left(k^{0}(t) ; u^{0}(t)\right)$, which maximizes the utility function (3) along the trajectories of the system (2) under the restriction (4) on the control variable $u$.

Problem analysis is conducted within the Pontryagin maximum for the problems over the infinite time interval $[3,4]$. Similar problems with smooth production function $y=f(k)$ are investigated in the papers $[10,11,12$, $16,17,18]$, where the existence of the unique optimal solution having the property of growth saturation is established. In order to apply the ideas proposed in these papers, we need to replace the non-smooth production function $y=f(k)$ with its estimate $\hat{y}=\hat{f}(k)$ that is differentiable for all $k \in \kappa$, and consider dynamic equation of the form (2). 


\section{PROBLEM ANALYSIS AND NUMERICAL EXAMPLE}

Solution of the Hamiltonian system is constructed according to the algorithm developed in the works $[1,12,16]$. Significantly, that there exists the unique steady state $P^{*}$ belonging to the domain $D_{2}$ of the transient control regime. Moreover, the steady state is a saddle point, since the Jacobian evaluated at the steady state has two real eigenvalues $\lambda_{1}$ and $\lambda_{2}$, where $\lambda_{1}<0$ and $\lambda_{2}>\rho>0$. According to the Grobman-Hartman theorem, the nonlinear system admits a trajectory the same as the linear system. This trajectory converges to equilibrium and is tangent to the eigenvector corresponding to the negative eigenvalue. This is a key fact for the algorithm, since it includes the integration of the Hamiltonian systems in the backward time starting from point $P_{\varepsilon}$ located at a vicinity of the steady state, in addition, the coordinates of point $P_{\varepsilon}$ are chosen in the direction of the eigenvector corresponding to the negative eigenvalue.

Numerical Algorithm includes the following steps: 1. Calculation of the steady state $P^{*}=\left(k^{*} ; \psi^{*}\right)$ as a solution of nonlinear algebraic equations; 2. Computation of eigenvalues $\lambda_{1}$ and $\lambda_{2}$ and eigenvectors $h_{1}=\left[h_{11} ; h_{12}\right]^{T}$ and $h_{2}=$ $\left[h_{21} ; h_{22}\right]^{T}$ of the Jacobian evaluated at the steady state $P^{*} ; 3$. For the chosen precision parameter $\varepsilon>0$, we select an initial point for the backward-time integration of the Hamiltonian system; 4. The backward-time integration of the Hamiltonian system (10) starting from the characteristic point $\left(k_{\varepsilon} ; \psi_{\varepsilon}\right)$. The integration is performed until one of two alternatives. If the integrated trajectory reaches the initial point $k_{0}$ in domain $D_{2}$ then the algorithm is stopped and the trajectory is built. If the integrated trajectory achieves sewing curves $\psi(t) \hat{f}(k(t))=1$ or $\psi(t) \hat{f}(k(t))=1 /(1-\bar{u})$, separating domains $D_{2}, D_{1}$ and $D_{2}, D_{3}$, respectively; 5 . The expansion of the integrated trajectory in the direct time and its time scaling.

The proposed algorithm ensures a good approximation of the optimal solution (see $[12,17])$. The production function $y=f(k)$ is a piecewise continuous function of the form:

$$
y=f(k)=\left\{\begin{array}{l}
\alpha_{1} k^{\beta_{1}}, k \leq k_{c} \\
\alpha_{2} k^{\beta_{2}}, k \geq k_{c}
\end{array} .\right.
$$

The identification procedure is carried out for the regression model: $\ln y=\beta_{1} \ln k+\ln \alpha_{1}+r \gamma\left(\ln k-\ln k_{c}\right)$, where parameter $r=0$ for $k<k_{c}$, and $r=1$ for $k \geq k_{c}, \alpha_{2}=\alpha_{1} k_{c}^{-\gamma}$, and $\beta_{2}=\beta_{1}+\gamma$. Econometric analysis performed by the real data provides the following estimates $\alpha_{1}=0,7466 ; \beta_{1}=0,8806 ; \alpha_{2}=1,9605 ; \beta_{2}=0,5137$. The determination coefficient $R_{2}$ equals to $R_{2}=0,9954$, this high value proves good fitness of the regression model. Next, we construct a smooth estimate of the production function $y=f(k)$, such that the approximation is a positive, increasing, smooth and concave function for all $k$. For this purpose, we design an asymptotic state observer that allows for constructing such an approximation that has smooth first and second derivatives, and uses only the continuous production function.

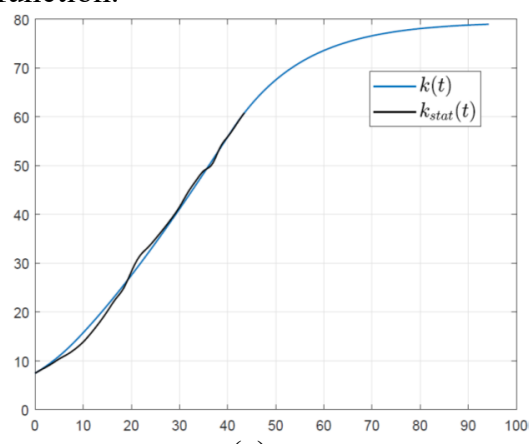

(a)

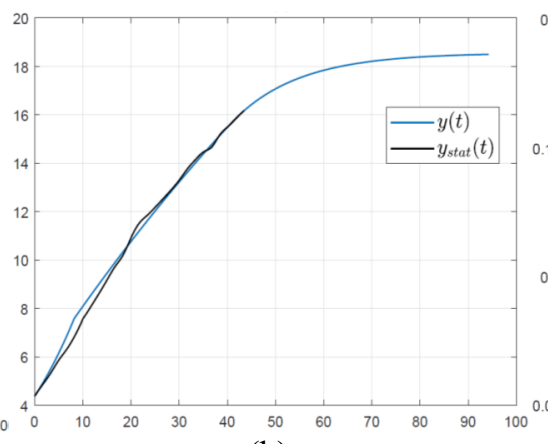

(b)

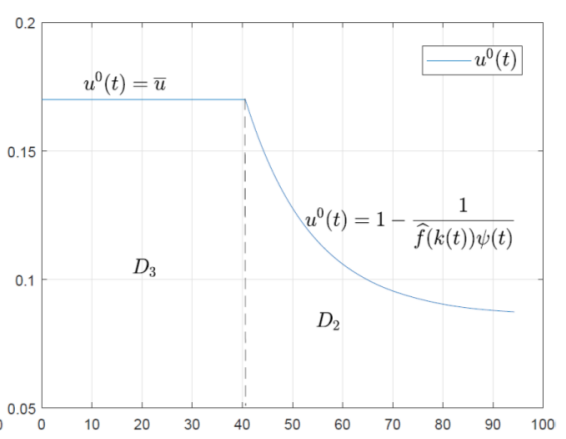

(c)

FIGURE 1. (a) Capital $k(t)$, optimal trajectory and statistical trends, (b) GDP $y(t)$, optimal trajectory and statistical trends, (c) Control $u^{0}(t)$.

The simulated trajectories of the capital $k(t)$, the output $y(t)$ and the investment plan $u^{0}(t)$ are depicted in Fig. 1, respectively, in comparison with the statistical data. The comparison shows that the synthetic trajectory adequately reflects trends of the real data. The graph of investments and the sewing curve show that, at the beginning, the optimal investment level stays at the intensive level $u=0,17$. Then, on the sewing curve, the switch happens from the intensive level to the transition level $u^{0}(t)$, which is implemented in domain $D_{2}$; finally, while time $t$ grows to infinity, the investment level monotonically decreases to the level $u^{*}=0: 0856$, determined by the steady state $P^{*}=$ $\left(k^{*} ; \psi^{*}\right)$. 


\section{CONCLUSION}

In the paper, the optimal control problem is investigated for the model of economic growth with the non-smooth production function in the presence of switching modes for basic model parameters. The switching modes can be detected by means of the classical econometric regression with dummy variables. The optimal control problem for the identified hybrid system is analyzed within the framework of the Pontryagin maximum principle adapted to problems with the infinite time horizon. The non-smooth production function is approximated with the function having the properties commonly inherent in production functions. The estimated production function is derived as a solution of the system (20), where the quality of approximation depends on the chosen eigenvalues of the matrix. These eigenvalues are determined numerically, such that the required conditions are satisfied. Application of the proposed method to the specific optimal control problem shows that the simulated trajectory adequately reflects trends of the real data, and the proposed algorithms can be used for forecasting future scenarios of growth.

\section{ACKNOWLEDGEMENTS}

Research is supported by the complex project of UbRAS No.18-1-1-10.

\section{REFERENCES}

[1] Ane, B.K., Tarasyev, A.M., and Watanabe, C. (2007). Construction of nonlinear stabilizer for trajectories of economic growth. Journal of Optimization Theory and Applications. 134(2). pp. 303-320.

[2] Arrow, K.J. (1985). Production and Capital. Collected Papers. 5. The Belknap Press of Harvard University Press, Cambridge, Massachusetts, London.

[3] Aseev, S.M. and Kryazhimskiy, A.V. (2007). The Pontryagin maximum principle and optimal economic growth problems, volume 257. Pleiades Publishing.

[4] Ayres, R., Krasovskii, A.A., and Tarasyev, A.M. (2009). Nonlinear stabilizers of economic growth under exhausting energy resources. IFAC-PapersOnLine.

[5] Ayvazyan, S.A. (2010). Econometric Methods. Magistr: INFRA-M.

[6] Crespo Cuaresma, J., Palokangas, T., and Tarasyev, A. (2010). Dynamic Systems, Economic Growth, and the Environment. Springer, Heidelberg, New York, Dordrecht, London.

[7] Crespo Cuaresma, J., Palokangas, T., and Tarasyev, A. (2013). Green Growth and Sustainable Development. Springer, Heidelberg, New York, Dordrecht, London.

[8] Grossman, G.M. and Helpman, E. (1991). Innovation and Growth in the Global Economy. MIT. Press.

[9] Krasovskii, A. and Tarasyev, A. (2008a). Conjugation of hamiltonian systems in optimal control problems. IFAC-PapersOnLine. 17(1), pp. 7784-7789.

[10] Krasovskii, A.A., Kryazhimskiy, A.V., and Tarasyev, A.M. (2008). Optimal control design in models of economic growth. Evolutionary Methods for Design, Optimization and Control. CIMNE, Barcelona. pp. 70-75.

[11] Krasovskii, A.A. and Taras'ev, A.M. (2007). Dynamic optimization of investments in the economic growth models. Automation and Remote Control. 68(10). pp. 1765-1777.

[12] Krasovskii, A.A. and Tarasyev, A.M. (2008b). Properties of hamiltonian systems in the pontryagin maximum principle for economic growth problems. Proc. of the Steklov Institute of Mathematics. 262(1). pp. 121-138.

[13] Pontryagin, L.S., Boltyanskii., V.G., and et al. (1962). The Mathematical Theory of Optimal Processes. Interscience, New York.

[14] Shell, K. (1969). Applications of pontryagins maximum principle to economics. Math. System Theory and Economics. 1. pp. 241-292.

[15] Solow, R.M. (1970). Growth theory: An exposition. Oxford University Press, New York.

[16] Tarasyev, A.M. and Usova, A.A. (2010). Construction of a regulator for the hamiltonian system in a two-sector economic growth model. Proc. of the Steklov Institute of Mathematics. 271(1). pp. 265-285.

[17] Tarasyev, A.M. and Usova, A.A. (2012). Stabilizing the hamiltonian system for constructing optimal trajectories. Proc. of the Steklov Institute of Mathematics. 277(1). pp. 248-265.

[18] Tarasyev, A.M. and Usova, A.A. (2015). Structure of the jacobian in economic growth models. IFACPapersOnLine. 2015. 48(25). pp. 191-196.

[19] Tarasyev, A.M. and Watanabe, C. (2001). Optimal dynamics of innovation in models of economic growth. Journal of Optimization Theory and Applications. 108(1). pp. 175-207. 